\title{
Precision Cosmic Ray physics with space-born experiment
}

\author{
Marco Incagli $^{\mathrm{a}}$ \\ Istituto Nazionale di Fisica Nucleare (INFN), Pisa, Italy
}

\begin{abstract}
More than 100 years after their discoveries, cosmic rays have been extensively studied, both with balloon experiments and with ground observatories. More recently, the possibility of mounting detectors on satellites or on the International Space Station has allowed for a long duration (several years) continuous observation of primary cosmic rays, i.e. before their interaction with the earth atmosphere, thus opening a new regime of precision measurements. In this review, recent results from major space experiments, as Pamela, AMS02 and Fermi, as well as next generation experiments proposed for the International Space Station, for standalone satellites or for the yet to come Chinese Space Station, will be presented. The impact of these experiment on the knowledge of Cosmic Ray propagation will also be discussed.
\end{abstract}

\section{Introduction}

Charged Cosmic Rays cover many orders of magnitude, both in flux and in energy range. Therefore many different techniques have been established to study them over the whole spectrum. In particular, large areas have to be covered in order to observe cosmic rays with energy above $10^{15} \mathrm{eV}$, where $\simeq 1$ particle $/ \mathrm{m}^{2} /$ year is expected ${ }^{1}$.

With current techniques, observations at such energies and above can only be performed on ground ${ }^{2}$.

On the other hand, particle fluxes at energies $1 \mathrm{GeV}-1 \mathrm{TeV}$ are of order 1 particle $/ \mathrm{m}^{2} /$ second and experiments having dimensions $\simeq 1 \mathrm{~m}^{3}$ can be used. This has allowed the deployment of some detectors in space, either as free-flyers - PAMELA, FERMIGLAST - or as payloads mounted on the International Space Station (ISS) - AMS02 and, in the near future, CALET, ISS-CREAM.

The clear advantage of such detectors, with respect to ground based ones, is that they are sensitive to the primary CR component, where by primary I mean "before interacting with the earth atmosphere", they can reach a much higher precision on the energy determination, in particular for electromagnetic particles, and on the chemical composition. Moreover, if equipped with a magnet, they are sensitive to anti-particles. On the other hand, due to the

\footnotetext{
a e-mail: marco.incagli@pi.infn.it

${ }^{1}$ Here I am referring to the integral flux. The flux of particular species (nuclei, leptons, ...) can vary by orders of magnitude, as we will see in the next sections.

2 A noticable exeption to this paradigma is the possibility of observing the fluorescence light, emitted by very energetic air showers $\left(>10^{20} \mathrm{eV}\right)$, from a telescope orbiting at an altitude of $\simeq 400 \mathrm{~km}$. Several such experiments have been proposed in the past - EUSO, JEM-EUSO, KLYPVE, OWL; as of now, only a small prototype (MINI-EUSO) has a good chance of being launched on the International Space Station in the near future.
} 
large cost of space missions, such detectors are limited both in mass and in geometrical dimensions, which results in a severe limitation in the energy range due to the steeply falling CR flux.

As an intermediate step between ground and space detectors, balloon experiments have to be mentioned. Flying at an altitude of $37 \mathrm{~km}$, with only $5 \mathrm{gr} / \mathrm{cm}^{2}$ of residual atmosphere above, these experiments share similar advantages as space ones, at a much lower cost. However balloon flights last at most 3 weeks, so the integration time is quite limited.

In this review I will focus on space experiments discussing the general characteristics (Sect. 2) with a specific reference to geometrical acceptance and background rejection (Sect. 3). I will then discuss the physics case and the recent results from the two major experiments focused on charged cosmic rays: PAMELA and AMS02 (Sects. 4 and 5). As there are specific presentations on photon physics, in these proceedings, I will not discuss here results from the FERMI experiment, although it will be mentioned while describing the general characteristics of space experiments. Finally I will briefly mention the detectors foreseen for the near or the more far future (Sect. 6).

\section{Space detectors}

Although several techniques can be (and have been) used for building or designing space detectors, three general classes of experiments can be identified:

1. magnetic spectrometers (à la AMS02);

2. pair-conversion telescopes (à la FERMI);

3. calorimeters (à la ATIC or CREAM, but also CALET, DAMPE or ISS-CREAM) with a possible enphasis on hadrons (nuclei) or leptons $\left(e^{ \pm}, \gamma\right)$ which can, on their turn, be more specialized on hadrons or on electromagnetic showers.

Of course, different combinations of the different techniques are possible.

\subsection{Magnetic spectrometers}

Spectrometers allow for the measurement of the particle momentum and of the sign of its charge. As a consequence, it is possible to have access to anti-particles, in particular to positrons and anti-protons, but also, at least in principle, to anti-deuteron or anti-helium nuclei.

Moreover, by combining the momentum with the measurement of the velocity $\beta$, performed either through Cherenkov or through Time Of Flight (TOF) techniques, it is possible to determine the nuclear mass number $A$ via the relation:

$$
A=\frac{R Z e}{m_{N} c \beta \gamma}
$$

where $m_{N}$ is the nucleon mass ${ }^{3}$, thus giving access to isotopes $\left({ }^{3} \mathrm{He},{ }^{4} \mathrm{He},{ }^{9} \mathrm{Be},{ }^{10} \mathrm{Be}, \ldots\right)$. A review of the techniques used for isotope identification requires a specific discussion of TOF or Cherenkov detectors, which goes beyond the scope of this generic introduction.

With current technologies, two solutions are possible for magnets: permanent magnets and superconducting magnets working at the superfluid helium temperature. Several interesting $R \& D$ are in progress to develop superconducting magnets operating at higher

\footnotetext{
${ }^{3}$ It is easy to write an expression in which the neutron and the proton masses appear explicitely instead of the average $m_{N}$. However, the error introduced by using this approximation is much smaller than the one due to the momentum or velocity measurements.
} 
temperatures ([1]). However these techniques have still to be fully developed, and then it will be necessary to test them for space. So this possibility will not be discussed any further.

Permanent magnets are heavy and their magnetic field is limited to values of $\simeq 1 \mathrm{kGauss}$; superconducting magnets are hard to operate in space and require a reservoir of Helium to be carried on board. For example, in the first project of the AMS02 experiment a dewar with 35001 of Helium was foreseen. With this amount of helium, the lifetime of the experiment was planned to be $\simeq 28$ months ([2]).

\subsection{Pair-conversion telescopes}

A pair-conversion telescope is a detector with a dedicated tracking stage having a thickness of 1-2 radiation lengths, normally obtained by interleaving silicon active material with tungsten layers, in which photons interact converting in an $e^{+} e^{-}$pair. The two leptons are precisely traced and their energy is measured by the calorimeter located below. So these detectors are mostly dedicated to precision $\gamma$ physics at energies which can be as low as $\simeq 1 \mathrm{MeV}$, which is the threshold energy for electron pair production. As a matter of fact, electrons produced with a momentum of few $\mathrm{MeV}$ undergo multiple scattering in the passive layers and their momentum cannot be effectively measured. So the lower energy limit is really $\simeq 100 \mathrm{MeV}$. An example of such an experiment is FERMI ([3]). To reach the $1 \mathrm{MeV}$ limit, a modified structure in which the passive layers are replaced by additional active silicon has been proposed in ([4]).

These type of detectors have an excellent angular resolution, or Point Spread Function (PSF). However the presence of the heavy tracker introduces some complexity in the system which has some impact in the Field Of View (FOV) and in the energy resolution.

\subsection{Calorimeters}

Calorimeter-based detectors are specialized in measuring the nuclear charge and the energy of electromagnetic showers. The limit on the total weight and dimensions does not allow to build hadronic calorimeters as on ground. However it is nevertheless possible to measure the energy of hadrons by using some special techniques, as described later for the ISS-CREAM experiment.

The main characteristic of calorimeters, however, is clearly to maximize the geometrical acceptance, by dedicating all the mass budget to the detector.

Comparing calorimeters to gamma-converters and to magnetic spectrometers, it is possible to note that the pointing capability for photons is $\simeq 0.5^{\circ}$, more than enough for anisotropy studies, and that, although not sensitive to anti-particles, calorimeters can accurately study the total $e^{+}+e^{-}$flux, thus being able to detect sharp variations in the spectrum induced by a potential DM source.

\section{Statistics vs. Acceptance and the issue of background}

The CR flux decreases very rapidsly with the energy. As an example, Fig. 1 shows the number of electrons $\left(e^{+}+e^{-}\right)$and of positrons expected above a given energy for several geometrical acceptances, indicated in the plot by different lines.

The two colored dashed and dotted lines approximately indicate the geometrical acceptances of FERMI and AMS02 experiments. The numbers represent the effective geometrical acceptance integrated over one year, where effective means that all inefficiencies, including dead time, trigger efficiency and so on, are accounted for. For an apparatus having the size of Fermi, which is currently the high energy space detector with the largest acceptance, at most 100 electron events per year are expected at energies $\mathrm{E}=2-3 \mathrm{TeV}$. This number rapidly 

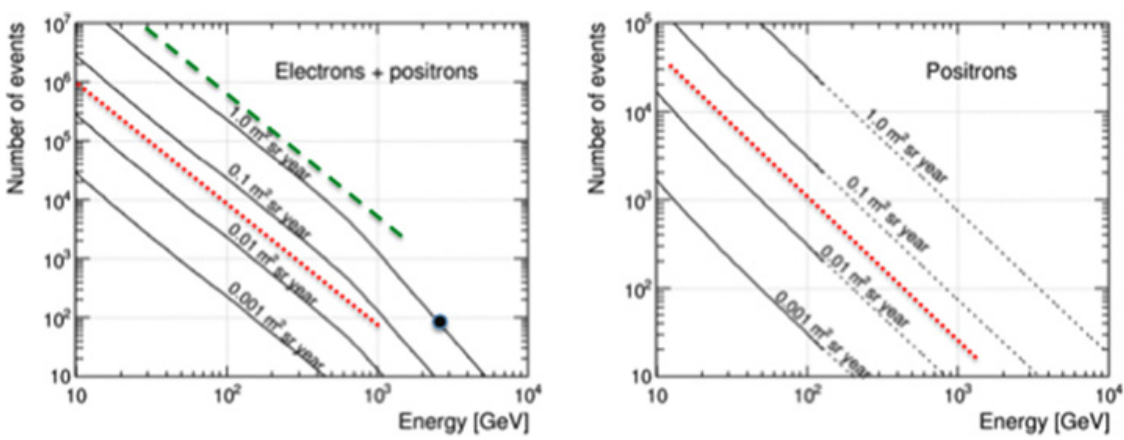

Figure 1. Number of events above a given energy (efficiency cuts not considered) for different geometrical exposures. The red dotted and the green dashed lines appreoximately represent the geometrical acceptance of the AMS02 and the FERMI experiment, respectively.

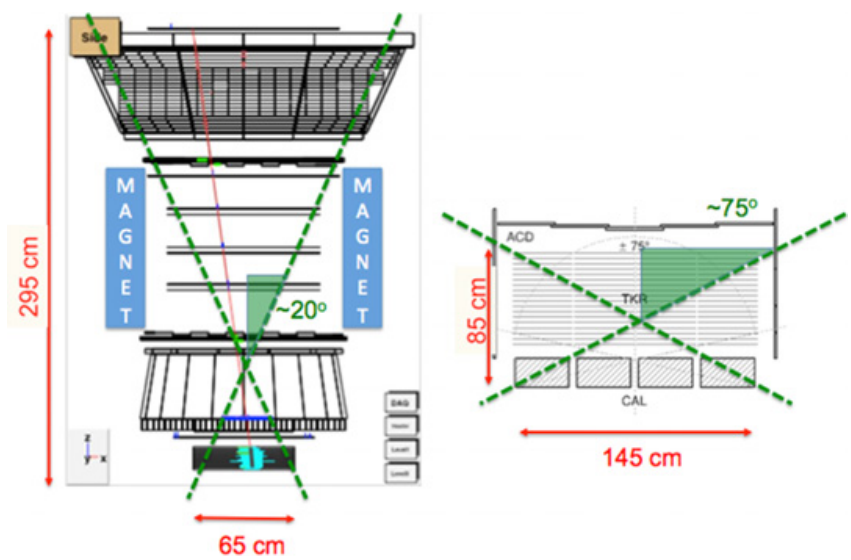

Figure 2. AMS02 and FERMI detectors in scale. Different dimensions and different geometrical acceptances are evident.

decreases for a detector whose FOV is limited by the presence of the magnet. Figure 2 shows the two detectors on the same scale, putting in evidence the different choices made.

However statistics is not the whole story. Different channels, in particular anti-particles, have fluxes which can be suppressed by several orders of magnitude with respect to the most common ones. Figure 3 shows the flux, as a function of energy, for some particle types with respect to protons. In this plot, three regions can be identified: ep-rejection, charge confusion and photons.

- ep-rejection: electrons suffer from a proton background of $10^{2}-10^{3}$, depending on the energy, while for positrons this background is $\simeq 10^{4}$; as a consequence, to measure the electron spectrum in the $\mathrm{TeV}$ region with a precision better than $10 \%$, an electronproton (ep) rejection of $10^{-4}-10^{-5}$ must be achieved, while still keeping a high signal efficiency to keep under control the statistical error.

- charge confusion: protons are approximately 4 orders of magnitude more abundant than anti-protons. Therefore the sign of the charge has to be determined with such a level of precision in order to avoid the charge confusion effect, i.e. the wrong sign reconstruction. 


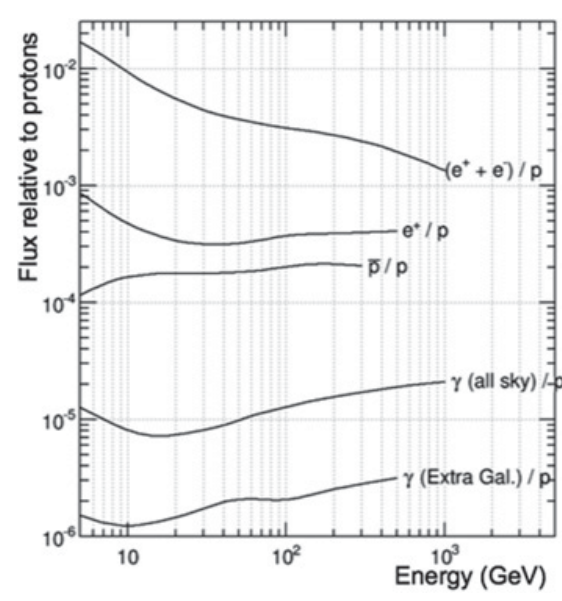

Figure 3. Cosmic ray flux as a function of energy relative to protons for several species.

- photons: to measure photons, a proton suppression factor of order ppm is required. All these constraints must be considered while designing experiments for space.

\section{The physics case: Cosmic Rays and Dark Matter search}

Many topics may be studied in space experiments. The two main ones are: Cosmic Rays (CR) origin and propagation and Dark Matter (DM) searches.

\subsection{Cosmic Rays origin and propagation}

The reference theoretical framework which is widely used to interpret CR nuclear data is based on the following main assumptions/approximations:

- CR diffusion is treated as isotropic: the interstellar diffusion tensor is assumed to be a scalar independent from rigidity, the so called diffusion coefficient $D$;

- the diffusion coefficient is assumed to be spatially uniform and (in most cases) to have a simple power-law dependence on the particle rigidity: $D(\rho) \propto \rho^{\delta}$;

- all primary nuclear species share the same single (or broken) power-law acceleration shape up to $E \simeq 10^{15} \mathrm{eV}$, the so called knee;

- CR sources are continuosly distributed in the Galactic disk.

With these approximations, the main experimental features of primary and secondary CRs are reasonably well reproduced (fluxes, $\bar{p} / p$ ratio, primaries/secondaries spectra, ...). However new and more precise experimental data are in tension with the predictions of this standard scenario and call for a more detailed and complete theoretical framework.

Among the observables which are in tension with the standard CR description, some of the ones which are relevant for space experiments are:

- p/He ratio: ATIC, CREAM and PAMELA found different spectral indexes for proton and Helium CRs. Preliminary AMS02 results seem to confirm this difference. This is hardly explainable in terms of Fermi acceleration theory, which does not differentiate between elements at ultra-relativistic rigidities. 
- CR spectra hardening: PAMELA found a sharp spectral breaking of $p$ and He spectra at the same rigidity $p_{\text {break }} \simeq 240 \mathrm{GeV}$. Although AMS02 preliminary data do not confirm this result, it surely allows for a better connection with CREAM data in the $10-500 \mathrm{TeV}$ energy range. Since a spectral breaking is not predicted by Fermi acceleration theory, this hardening may either require to abandon the continuos source distribution limit and/or to introduce major changes in the way CR propagation is treated.

- anisotropy and secondary to primary ratio: an anisotropy in CR arrival direction is expected, with a larger contribution from the galactic center with respect to the external part of the galaxy. Models which predict a diffusion coefficient $\delta>0.5$, favored by recent $\mathrm{B} / \mathrm{C}$ ratio data, severely overpredict this anisotropy.

These observational issues call, on one hand, for an improved description of the CR production and propagation and, on the other hand, for more experimental data with improved resolution to further constraint the theory. The main role of space experiments, in this respect, is there capability of discriminating the different components by measuring the $Z$ of nuclei by $d E / d x$ in different materials, tipically scintillators or tracker silicon layers, or by other techniques (e.g. the amount of photons observed in the rings produced by particle crossing radiators in RICH counters).

\subsection{Dark Matter identification}

There is now a overwhelming experimental evidence of the presence of a large component of a new type of particles, generally called Dark Matter (DM), whose mass makes up $\simeq 80 \%$ of the mass budget of the universe. Although there is no evidence of the energy scale of this new form of matter, which could range from invisible to macroscopic objects, there is a particular solution which has several attractive features: the Weakly Interacting Massive Particles (WIMP). There is a singular coincidence between the parameters of the Standar Model of particle physics and of the Cosmological Model to provide valid DM candidates at the electroweak scale $(\simeq 1 \mathrm{TeV})$ with a cross section typical of weak processes.

These particles are being studied in different processes:

- the annihilation, or indirect, channel, in which to DM particles annihilate to produce two standard ones. This channel is the relevant one for space experiments;

- the interaction, or direct channel, is pursued in underground laboratories which look for the interaction of the WIMP wind, caused by the earth motion in the solar system, and in the galaxy in general, with ordinary ultra-pure matter;

- the production in collisions of Standard Model particles, as studied in colliders.

The annihilation channel can be detected by an excess of particles with respect to the ones produced in standard Cosmic Rays. It is normally expected that the DM annihilation provides a particle-antiparticle pair, therefore, being antiparticles much rarer than particles in CRs, cosmic rays, the eventual excess would be more relevant in the positron or the antiproton channel than in the correspondants particles.

For this reason, it was proposed in the 90s that monoenergetic positrons from halo annihilations could be a significant and distinctive signal for massive dark-matter particles [7]. The signature would be an increase in the positron fraction $\left(e^{+} /\left(e^{+}+e^{-}\right)\right)$with respect to the one expected from the interaction of primary cosmic rays with the interstellar medium. To detect positrons a magnetic detector is required, which led to the construction and set in orbit first of the PAMELA experiment, on a dedicated satellite, and then of AMS02, installed on the ISS. 


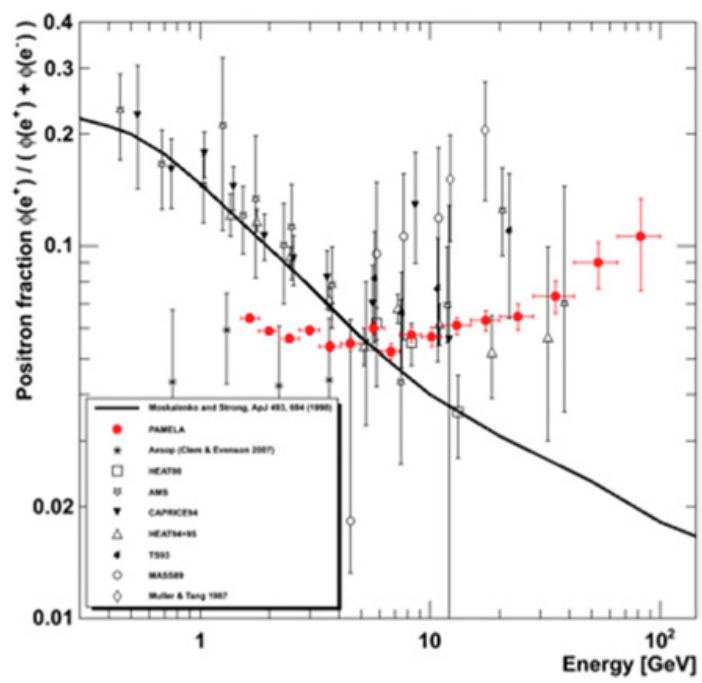

Figure 4. Positron fraction measured by PAMELA experiment.

\section{The magnetic spectrometers: PAMELA and AMS02}

The two detectors are described in detail in several publications (e.g. [5, 6]). The main structure is a permanent magnet with a dipole field, in both cases with intensity $\simeq 1 \mathrm{kG}$, equipped with a set of silicon tracking layers, followed by an electromagnetic calorimeter having a thickness of $\simeq 17 X_{0}\left(X_{0}=\right.$ radiation length $)$. Both detectors are also equipped with a Time Of Flight trigger system and a set of Anti-coincidence counters to reject tracks entering the apparatus from the sides. AMS02, in addition, has a Transition Radiation Detector, for electron-proton (or positron-proton) rejection, and a Ring Image Cherenkov Counter, which can measure the particle velocity $\beta$ and can provide information on isotope composition. However the main difference between the two detectors are the dimensions and, as a consequence, the Geometrical Acceptance: PAMELA weighs $\simeq 500 \mathrm{~kg}$ and has an acceptance $A \simeq 20 \mathrm{~cm}^{2}$ sr, while AMS02 payload weighs $\simeq 7$ tons $^{4}$ and its effective geometrical acceptance (i.e. after selection) is of $\simeq 450 \mathrm{~m}^{2}$ sr. PAMELA was launched in June 2006; AMS02 in May 2011.

\subsection{Positron excess and anti-proton flux}

The first clear evidence of an excess of positrons in the flux of Cosmic Rays was published by PAMELA in April 2009 on Nature ([5]) and it is represented in Fig. 4. The observed spectrum is incompatible with a secondary production, which is expected to smoothly decrease as in the solid line of Fig. 4. Therefore, either a significant modification in the acceleration and propagation models for cosmic rays is needed, or a primary component is present.

A modification in the propagation of secondary cosmic rays has been proposed by several authors ([8,9]). The proposed effect, which could explain the observed "excess", is that a fraction of the secondary positrons are produced inside the same astrophisical source which accelerate the primaries, such as supernova remnants. The flux of positrons produced at the shock is naturally harder than the standard one and could explain the rise in the observed

\footnotetext{
${ }^{4}$ Almost half of this weight is due to the support system which connects the detector to the ISS.
} 
positron fraction. The same mechanism, however, predicts a similar effect also on the ratio $\bar{p} / p$ and $B / C$ (Boron over Carbon), excess which, at the moment, has not been observed ${ }^{5}$.

Alternatively, the excess could be due to the existence of a primary source which, tipically, produces $e^{+}, e^{-}$pairs. A very intriguing possibility is that these pairs are produced by the annihilation of DM particles, as suggested in [7]. However there are also more standard scenarios in which positrons are produced by a nearby astrophysical source; for example a pulsar could emit energetic photons that, interacting with the strong magnetic field surrounding the neutron star, decay in an $e^{+} e^{-}$pair [12].

Although this excess generated a lot of excitement in the community, in view of the potential DM explanation, the measurement of the anti-proton flux by the same collaboration showed no excess with respect to standard secondary production [13]. While this result is well compatible with the pulsar hypothesis, a well behaving DM particle would instead produce a sizeable proton-antiproton flux, unless some ad hoc mechanism is invoked. An additional difficulty in the DM explanation is the magnitude of the effect, as it exceeds what is expected by the thermal cross section requiring sizeable boost effects. Several ways out have been proposed (see, e.g., [14] or [15]), but to discriminate between the different possible explanations, more precise measurements extending at higher energies are necessary.

\subsection{The AMS02 experiment}

A new measurement of the positron and electron fluxes, as well as of the positron fraction, has been recently performed by the AMS02 experiment with a much higher statistics. Details are given in [16]. Here we note that positron events suffer from two different backgrounds:

1. ep-rejection: protons which are wrongly reconstructed as electromagnetic showers (protons are 4 orders of magnitude more abundant than positrons);

2. charge confusion: electrons whose charge has been wrongly identified in the tracker and are reconstructed as positrons.

In AMS02 electrons are distinguished from protons by three different, and in large part independent, detectors:

- the Transition Radiation Detector (TRD) located on top of AMS02 measures the $\delta$ - electrons emitted by relativistic particles when crossing the surface between materials with different dielectric constant. This effect is summarized in a likelihood estimator, as shown in Fig. 5(a);

- the ECAL measures the 3D shower shape and, by using a multivariate approach, distinguishes electrons from protons as shown in Fig. 5(b);

- finally, by comparing the total energy deposited in ECAL with the tracker momentum, leptons peak at $E / p=1$, while hadrons are shifted to zero, having a sizable leakage in the ECAL (Fig. 5(c).

Details of the analysis are in [16]. The resulting positron fraction is shown in Fig. 6, which extends the previous measurements up to $500 \mathrm{GeV}$ (upper edge of last bin). The excess is confirmed with high precision; moreover, a clear flattening in the fraction occurs starting from $\simeq 200 \mathrm{GeV}$. This behaviour is compatible both with the pulsar and with the DM explanation, as in both cases a cutoff is expected. In the first case the cutoff is due to the energy of the emitted photons, while in the second one the upper limit is determined by the DM mass. A

${ }^{5}$ See, however, [11] for a recent review which includes recent preliminary AMS02 data on $B / C$. 
a)

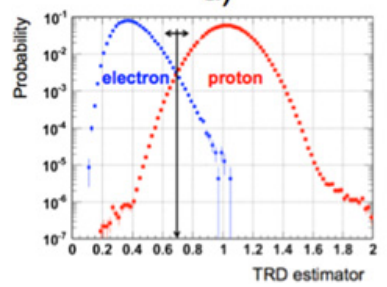

b)

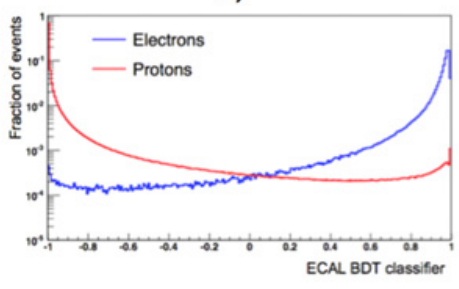

c)

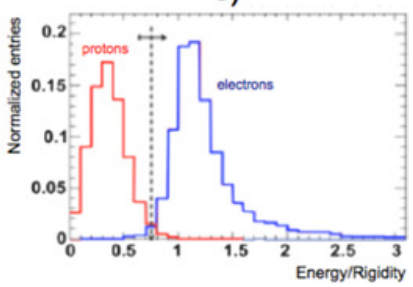

Figure 5. Electron-proton rejection in AMS02 through a) Transition Radiation Detector b) Electromagnetic Calorimeter c) Energy/Rigidity ratio.

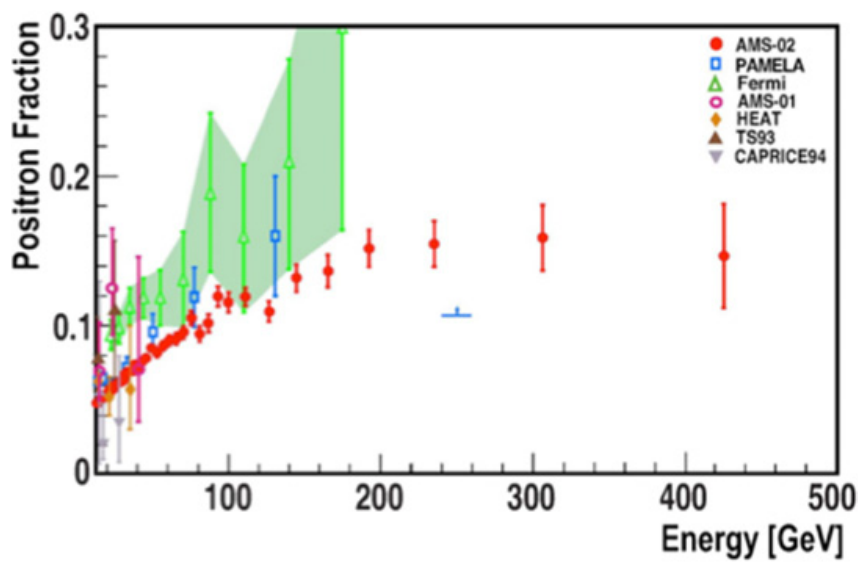

Figure 6. Positron fraction measured by AMS02 for energies above $10 \mathrm{GeV}$, where the fraction starts to increase. AMS02 shows, for the first time, a clear flattening of the fraction starting at $200 \mathrm{GeV}$.

possibility to discriminate between the two scenarios is shown in Fig. 7 in which the AMS02 results are extrapolated under two different models: typical DM-like models show a sharper cutoff with respect to pulsar-like ones. However this conclusion is not so firmly established (see, for example, [17]).

The separate fluxes have also been measured [18] and are shown in Fig. 8, where the positrons have been scaled up by a factor 10 in order to be compared with the electrons (positron scale on the right axis). A comparison between the two histograms shows how the effect of this eventual primary source has a different impact on the two components. Due to the higher statistics, the electron flux has been extended up to $700 \mathrm{GeV}$.

Although a more detailed information on the electron and positron spectrum is certainly useful, only a multi-messenger approach may provide sufficient information to disantangle among the different explanations. On this respect, several other results are expected from the AMS02 collaboration:

- proton and helium flux

- B/C, C/O and B, C, O fluxes

- anti-protons

- light nuclei and Nitrogen $(\mathrm{Li}, \mathrm{Be}, \mathrm{N})$

- photons

- heavier nuclei

- isotopes $\left({ }^{3} \mathrm{He} /{ }^{4} \mathrm{He}\right.$ and $\left.{ }^{10} \mathrm{Be} /{ }^{9} \mathrm{Be}\right)$ 


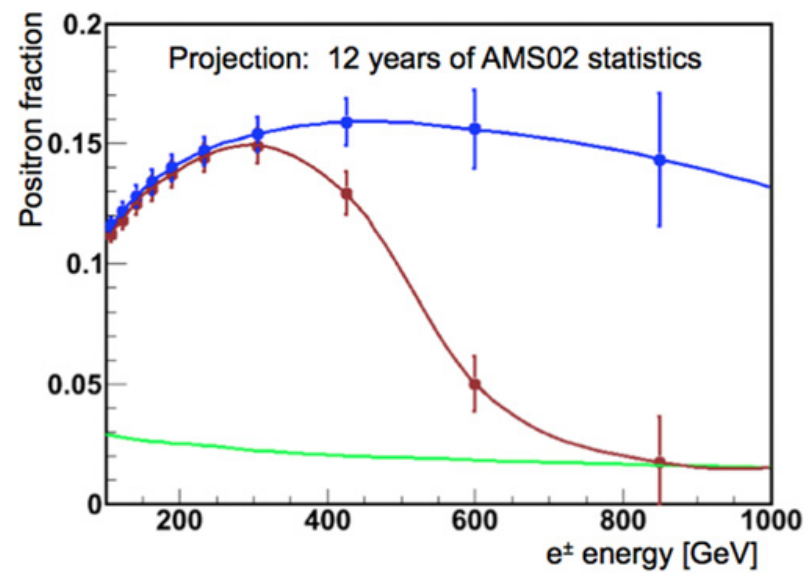

Figure 7. Projected statistical sensitivity of AMS02 experiment after 12 years of data taking. The lower, green line represents the expected background from secondaries. The brown middle line includes a DM contribution with mass $700 \mathrm{GeV}$; the upper blue line represents a Pulsar like model. These models are only representative.

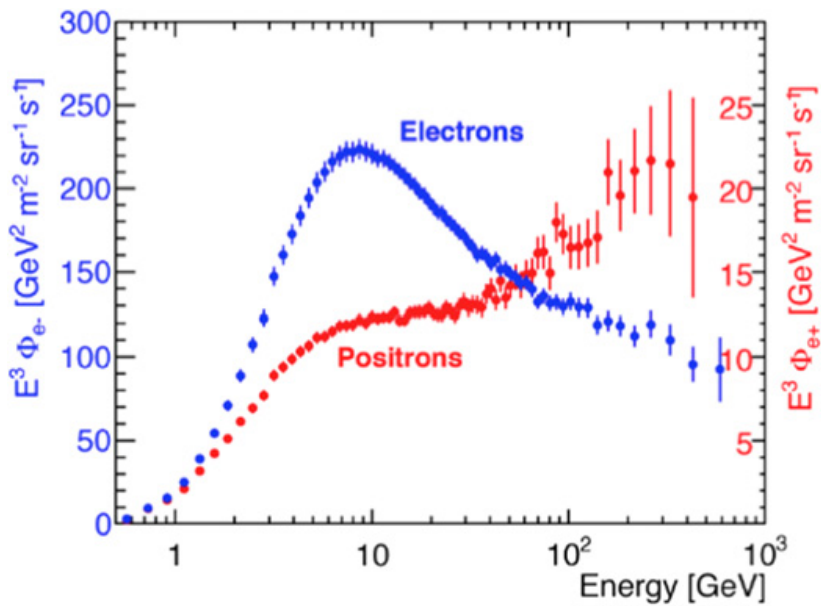

Figure 8. AMS02 flux for electrons (left axis) and positron (right axis).

- anti-D

- anti-He.

All these measurements are useful either as a direct probe of DM, as anti-protons and, possibly, anti-deuteron, or as a constraint to the propagation of $\mathrm{CR}$ in the insterstellar medium, as $\mathrm{B} / \mathrm{C}$ or as the isotope ratio.

\section{Future experiments}

\subsection{Near future}

Within 2017, three more space experiments are foreseen, all of calorimetric type: CALET [19], ISS-CREAM [20], DAMPE [21]. 
The first two will be mounted in 2015/2016 on the Japanese module of the International Space Station, while DAMPE is supposed to be launched at the end of 2016 on board of a chinese satellite.

CALET and DAMPE have very similar characteristics: the main detector is a thick electromagnetic calorimeter of $\simeq 30 X_{0}$, with very good energy resolution $(\simeq 1 \%$ at $1 \mathrm{TeV})$, pointing capability ( 0.5 degrees) and an excellent ep-rejection (at least $10^{-4}$ ), with a heavy tracker in front in which photons can convert. In CALET the tracker active material is made of $1 \mathrm{~mm}$ scintillating fibers, so the main focus is on the identification of electromagnetic and hadronic showers, while DAMPE uses silicon wafers, with more emphasis on photon pair production. Both detectors have very good $\mathrm{Z}$ discrimination capabilities. The geometrical acceptance is $0.1-0.2 \mathrm{~m}^{2} \mathrm{sr}$, thus a factor of 4 larger than AMSO2, but still an order of magnitude smaller than FERMI.

ISS-CREAM, instead, is a modified version of the CREAM experiment, who had six succesful antartic flights on aerostatic balloons between the years 2004 and 2010, for a cumulative exposure of 162 days. In spite of the success of the balloon missions, the experiment integrated less than half a year of equivalent data for a space mission, therefore the possibility of installing the apparatus on the ISS would boost the physics potential, at least in terms of accumulated statistics. The CREAM experiment extends the direct measurement of cosmic-ray composition to energies of hundreds of $\mathrm{TeV}$, at which CRs are capable of generating gigantic air showers which have been observed on the ground, thereby providing calibration for indirect measurements. A specific technique, which uses a preshower carbon detector half interaction length thick to induce hadronic cascades, is used to reach such energies with a thin calorimeter (thin in terms of hadronic showers). So, differently from previous experiments, this one is more focused on the precise measurements of the energy dependance of elemental spectra at the highest energies.

\subsection{Medium term experiments}

\subsubsection{GAMMA-400}

GAMMA-400 [22] is a russian-italian experiment which will fly on board of the russian Navigator spacecraft. The experiment will be initially installed on a highly elliptical orbit (with apogee $300.000 \mathrm{~km}$, perigee $500 \mathrm{~km}$ and an inclination of $51.4^{\circ}$ ), with 7 days orbital period; the orbit will then be gradually changed, in 5 months, to a circular one with a radius of $200.000 \mathrm{~km}$ radius. At such an orbit the Earth will not cover a significant fraction of the sky, as is usually the case for the Low Earth Orbit (LEO) of the experiments mentioned so far. Also the geomagnetic effect on charged particles will become negligible, which allows for a clean study of the low energy part $(E<10 \mathrm{GeV})$ of the spectrum.

The launch is foreseen for 2020. The project started with the main goal of improving FERMI results on photon physics. Therefore the apparatus has a converting tracker and a calorimeter having an energy resolution of $\simeq 1 \%$ at $1 \mathrm{TeV}$. To reach this energy resolution, the calorimeter has to be thicker than the FERMI one, with the consequence that the geometrical acceptance is almost a factor of 2 lower.

However, the project has been further developed and now the proposed calorimeter uses the CaloCube technique [23], which allows to use not only the upper face but also the lateral ones to detect CRs, with the consequence that the geometrical acceptance is increased by a factor 5 , at least for charged particles.

The introduction of a highly segmented homogeneous calorimeter made with CsI(Tl) cubes, with improved energy resolution and extended geometrical factor, coupled to the improvement of the converter-tracker structure make GAMMA-400 an excellent dual 
instrument, optimized both for photons (in the $100 \mathrm{MeV}-1 \mathrm{TeV}$ energy range) and charged cosmic rays (electrons up to $10 \mathrm{TeV}$ and high energy nuclei up to the knee region).

\subsubsection{The chinese space station}

China has recently announced an ambitious space program timetable with the goal of establishing its first space station around 2022. As part of this program, a major space experiment for Cosmic Ray detection is foreseen. Two possible designs are currently under study: a calorimetric detector surrounded by a pair-conversion tracker, similar in many aspects to GAMMA-400 but with a wider geometrical coverage, and a magnetic spectrometer.

The calorimetric apparatus option is more advanced, with a collaboration already building up around the design of HERD (High Energy cosmic Radiation Detection), while the magnetic spectrometer option, possibly an AMS03 mission, is still very preliminar.

Although at the moment it is too preliminary to make detailed comments about these possible detectors, it is evident the potentiality of such an observatory.

\section{Conclusions}

Many important and useful informations can be acquired by studying cosmic rays, both on their origin and propagation mechanism but also, possibly, on new physics, in particular Dark Matter.

Due to the large spread in energy and in flux, many different techniques have been developed. Among these, in the recent years space experiments have been characterized by the unique possibility of studying with great care the energy range from few hundred $\mathrm{MeV}$ up to hundreds of $\mathrm{TeV}$, at least for protons.

Space experiments have the unique opportunity of measuring anti-particles and isotopes, and have a greatly improved capability of discriminating different nuclear charges $Z$. Several important experiments are still collecting data: PAMELA, which is almost at the end of its life after 8 years of glorious mission, AMS02 and FERMI. Many new experiments have been proposed or are being built for the near and for the far future.

Only through a comparison of many different channels, the so-called "multi-messenger" approach, it will be possible to constraint the propagation of standard CRs and thus put severe limits on, or exclude some models of, Dark Matter.

\section{References}

[1] A. Ballarino 2010, J. Phys.: Conf. Ser. 234032003 ; "World-record current in the MgB2 superconductor", CERN Bulletin 16-17, April 2014

[2] AMS collaboration, "Upgrade of the Alpha Magnetic Spectrometer (AMS-02) for long term operation on the International Space Station (ISS)", Nucl. Instrum. Meth. A 654 (2011) 639-648

[3] W. B. Atwood et al., 2009 ApJ 697, 1071

[4] A. Morselli et al., Nuclear Physics B Proc. Supp. 239-240 (2013) 193-198

[5] Pamela collaboration, Nature 45B (2009) 607

[6] M. Aguilar et al., Phys. Rev. Lett. 110, 141102 (2013)

[7] M. S. Turner, F. Wilczek, Phys. Rev. D 42 (1990) 1001

[8] P. Blasi, Phys. Rev. Lett. 103, 051104 (2009)

[9] M. Ahlers, P. Mertsch, S. Sarkar, Phys. Rev. D 80, 123017 (2009)

[10] P. Blasi, P. Serpico, Phys. Rev. Lett. 103, 081103 (2009) 
[11] P. Mertsch, S. Sarkar, Phys. Rev. D 90 (2009) 061301

[12] I. Buesching et al., Astrophys. J. 78 (2008) L39

[13] Pamela Collaboration, PRL 105 (2010) 121101

[14] M. Cirelli et al., Nucl. Phys. B 813, 1 (2008)

[15] I. Cholis et al., Phys. Rev. D 80, 123518 (2009)

[16] AMS collaboration, Phys. Rev. Lett. 113, 121101 (2014)

[17] G. Bertone, M. Lattanzi, M. Pato, JCAP 1012 (2010) 020

[18] AMS collaboration, Phys. Rev. Lett. 113, 121102 (2014)

[19] Journal of Physics: Conference Series 409 (2013) 012026

[20] E. S. Seo et al., "Cosmic Ray Energetics And Mass (CREAM) for the ISS JEM-EF," the 2013 conference on TeV Particle Astrophysics (TeVPA) 2013

[21] J. Chang, in The 7th international workshop "Dark Side of the Universe (DSU 2011)" (2011)

[22] Nuclear Physics B (Proc. Suppl.) 239-240 (2013) 204

[23] O. Adriani et al., Development of a 3-D cubic crystal calorimeter for space: CaloCube, Proceedings of the International Conference on Calorimetry for the High Energy Frontier (CHEF 2013) 